\begin{tabular}{|c|c|c|c|c|c|c|}
\hline \multirow{4}{*}{ Impact Factor: } & ISRA (India) & $=3.117$ & SIS (USA) & $=0.912$ & ICV (Poland) & $=6.630$ \\
\hline & ISI (Dubai, UAE & $=0.829$ & РИНЦ (Russia & $=0.156$ & PIF (India) & $=1.940$ \\
\hline & GIF (Australia) & $=0.564$ & ESJI (KZ) & $=8.716$ & IBI (India) & $=4.260$ \\
\hline & JIF & $=1.500$ & SJIF (Morocco & $=5.667$ & OAJI (USA) & $=0.350$ \\
\hline
\end{tabular}

\section{SOI: $1.1 /$ TAS DOI: $10.15863 /$ TAS \\ International Scientific Journal \\ Theoretical \& Applied Science}

p-ISSN: 2308-4944 (print) e-ISSN: 2409-0085 (online)

Year: 2019 Issue: $08 \quad$ Volume: 76

Published: $05.08 .2019 \quad$ http://T-Science.org
QR - Issue

QR - Article
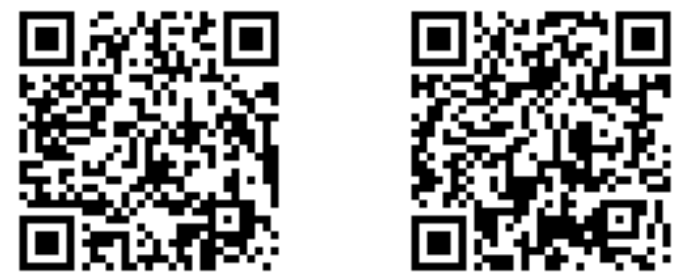

Ismoil Ibrohimovich Safarov

Tashkent Institute of Chemistry and Technology Doctor of Physical and Mathematical Sciences, Professor to department of Advanced Mathematics, Uzbekistan, safarov54@mail.ru

Nurillo Raximovich Kulmuratov Navoi State mining Institute Senior Lecturer to Department of Technology Engineering, Uzbekistan nurillo.kulmuratov.64@mail.ru

Anvar Ruzimov

Tashkent Institute of Chemistry and Technology Senior Lecturer to Department of Advanced Mathematics, Uzbekistan

\title{
INFLUENCE OF DYNAMIC DAMPENER ON DISTRIBUTIONS OF AREAS OF PARAMETRIC RESONANCE OF VISCOELASTIC MECHANICAL SYSTEMS WITH PERIODICALLY VARIABLE RIGIDITY
}

Abstract: We study the distribution of regions of parametric (and Raman) resonances in viscoelastic mechanical vibrational systems with periodically changing stiffness when a dynamic absorber is introduced into the system. It was shown that in this case two regions of simple and one combinational resonance are obtained instead of one region of parametric resonance in a system without a quencher.

Key words: viscoelastic body, resonance, absorber, parametric oscillations, stiffness, mass, damping coefficient.

Language: Russian

Citation: Safarov, I. I., Kulmuratov, N. R., \& Ruzimov, A. (2019). Influence of dynamic dampener on distributions of areas of parametric resonance of viscoelastic mechanical systems with periodically variable rigidity. ISJ Theoretical \& Applied Science, 08 (76), 1-6.

Soi: http://s-o-i.org/1.1/TAS-08-76-1 Doi: crossef https://dx.doi.org/10.15863/TAS.2019.08.76.1

Classifiers: Mechanics and machine construction.

\section{ВЛИЯНИЕ ДИНАМИЧЕСКОГО ГАСИТЕЛЯ НА РАСПРЕДЕЛЕНИЙ ОБЛАСТЕЙ ПАРАМЕТРИЧЕСКОГО РЕЗОНАНСА ВЯЗКОУПРУГИХ МЕХАНИЧЕСКИХ СИСТЕМЫ С ПЕРИОДИЧЕСКИ ИЗМЕНЯЮЩЕЙСЯ ЖЁСТКОСТЬЮ}

Аннотация: Изучается распределение областей параметрического (и комбинационного) резонансов 8 вязкоупругих механических колебательных систем с периодически изменяющейся жесткостью при введении в систему динамического гасителя. Показано, что при этом получаются две области простого и одна комбинационного резонансов вместо одной области параметрического резонанса в системе без гасителя.

Ключевые слова: вязкоупругая тела, резонанс, гаситель, параметрическая колебания, жесткость, масса, коэффициент демпфирования. 


\begin{tabular}{|c|c|c|c|c|c|c|}
\hline \multirow{4}{*}{ Impact Factor: } & ISRA (India) & $=3.117$ & SIS (USA) & $=0.912$ & ICV (Poland) & $=6.630$ \\
\hline & ISI (Dubai, UAI & $=0.829$ & РИНЦ (Russia & $=\mathbf{0 . 1 5 6}$ & PIF (India) & $=1.940$ \\
\hline & GIF (Australia) & $=0.564$ & ESJI (KZ) & $=8.716$ & IBI (India) & $=4.260$ \\
\hline & JIF & $=1.500$ & SJIF (Morocce & $=5.667$ & OAJI (USA) & $=0.350$ \\
\hline
\end{tabular}

Введение.

Большинство наблюдаемых в природе и технике процессов являются колебательными. В технике, особенно в машиностроении, широко применяют также термин вибрация, под которым понимают механические колебания относительно малой амплитуды и не слишком низкой частоты. В настоящее время наиболее простым, доступным и распространённым виброизолирующим средством остаются упругие элементы. На достаточно высоких частотах они обеспечивают отражение большей части колебательной энергии обратно в источник и тем лучше, чем ниже их жёсткость. В области низких частот требования к величине жёсткости, как правило, совсем иные. Они определяются статической нагрузкой, её изменениями при наклоне, а также инерционными силами при разгоне и торможении на движущихся объектах, ударами, центровкой механизма и прочими эксплуатационными условиями. Неоднозначны и требования к демпфированию: малая его величина полезна для виброизоляции, а в диапазоне низких частот порождает (при наличии вынуждающих) [1,2,3,4].

Виброизоляция - метод вибрационной защиты посредством устройств, помещаемых между источником возбуждения и защищаемым объектом [5]. Действие виброизоляции сводится к ослаблению связей между источником и объектом. При этом уменьшаются динамические воздействия, передаваемые объекту. Применение виброизоляции для защиты аппаратуры в широком диапазоне частот вынуждающих колебаний, как правило, не приводят к положительным результатам, т.к. эффективность виброизоляции на собственных частотах систем виброизоляции резко снижается [6,7]. Частотная отстройка применяется для устранения резонансных колебаний. Это обычно достигается за счет повышения значений собственных частот колебаний конструкции $[8,9,10]$, которая должна

не менее чем в 1,3 и более раз превышать частоты вынуждающих колебаний. Частотную отстройку наиболее часто обеспечивают за счет увеличения жесткости конструкции путем уменьшения площади, повышения жесткости крепления, увеличением толщины и количества точек крепления основания ячейки, установкой ребер жесткости и каркасов. Этот способ целесообразно применять, когда диапазон частот действующих вибраций не превышает 300-400 Гц, в крайнем случае, 500Гц. Было установлено, что при превышении этого диапазона устранить резонансные колебания невозможно без существенного, как правило, недопустимого, увеличения массы и габаритных размеров ячеек.

\section{Постановка задачи параметрического колебания и методики решения.}

Для выявления качественной картины распределения областей параметрического и комбинационного резонанса при присоединении динамического гасителя рассмотрим простейшую колебательную систему без трения, приведенную на рис.1. Здесь $m_{1}$ - масса основной системы, $c_{1}(1-\gamma \cos \omega t)$ - периодически изменяющаяся жесткость подвески основной системы, $\gamma$ коэффициент модуляции, малая величина, $m_{2}$ масса гасителя, $c_{2}$ - жесткость пружины гасителя, $\omega$ - частота пульсаций. Периодическое изменение жесткости подвески может быть обусловлено, например, наличием переменного магнитного поля (явление магнитострикции). Пусть $m_{2}<<$ $m_{1}$. Фактически предлагаемая схема представляет собой систему с двумя степенями свободы, и к ней применимы все выводы общей теории линейных дифференциальных уравнений с периодическими коэффициентами, но особенностью здесь является настройка и малая масса гасителя, что дает специфическую систему дифференциальных уравнений с малым параметром.

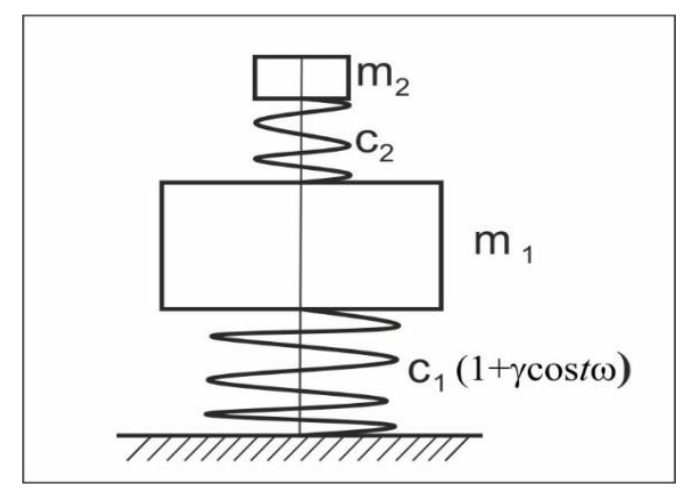

Рис.1. Расчетная схема.

Пользуясь принципом Даламбера для схемы на рис.1, получаем дифференциальные уравнения движения:

$$
\left\{\begin{array}{l}
m_{1} \ddot{x_{1}}+\tilde{c}_{1}(1-\gamma \cos \omega t) x_{1}-\tilde{c}_{1}\left(x_{2}-x_{1}\right)=0 \\
m_{2} \ddot{x_{2}}+\tilde{c}_{2}\left(x_{2}-x_{1}\right)=0
\end{array}\right.
$$




\begin{tabular}{|c|c|c|c|c|c|c|}
\hline \multirow{4}{*}{ Impact Factor: } & ISRA (India) & $=3.117$ & SIS (USA) & $=0.912$ & ICV (Poland) & $=6.630$ \\
\hline & ISI (Dubai, UAI & $=0.829$ & РИНЦ (Russia & $=0.156$ & PIF (India) & $=1.940$ \\
\hline & GIF (Australia) & $=0.564$ & ESJI (KZ) & $=8.716$ & IBI (India) & $=4.260$ \\
\hline & JIF & $=1.500$ & SJIF (Morocce & $=5.667$ & OAJI (USA) & $=0.350$ \\
\hline
\end{tabular}

$\tilde{c}_{\kappa} f(t)=c_{0 \kappa}\left[f(t)-\int_{-\infty}^{t} R_{c \kappa}(t-\tau) f(\tau) d \tau\right]$,

$(\kappa=1,2), x_{1}, x_{2}$-перемещения массы $m_{1}$ и $m_{2}$ относительно положения статического равновесия в неподвижной системе координат, $f(t)$ - произвольная функци времени; $R_{c k}(t-\tau)$ - ядро релаксаци, $c_{0 \kappa^{-}}$мгновений коэффициент жесткости.

При $\gamma=0$ система уравнений (1) представляет собой хорошо изученные уравнения движения системы с динамическим гасителем $(0 \leq \gamma \leq 1)$ [8]. При отсутствии динамического гасителя $m_{2}=0$ из (1) имеем одно дифференциальное уравнение

$$
m_{1} \ddot{x}_{1}+\tilde{c}_{1}(1-\gamma \cos \omega t)=0
$$

детально исследованное в литературе и известное под названием уравнения Матье. Непосредственно, или после некоторых упрощений, уравнением (2) описывается движение в ряде характерных задач механики,

например, колебания маятника с периодически изменяющейся. длиной, простейшая модель динамической устойчивости стержня (2) и др.

Присоединяя к названным системам вспомогательную массу на пружине (динамический гаситель), приходим к уравнениям типа (1). Перепишем систему (1) в виде

$\left\{m_{1} \ddot{x}_{1}+\left(\tilde{c}_{1}+\tilde{c}_{2}\right) x_{1}-\tilde{c}_{2} x_{2}=\varepsilon \tilde{c}_{1} \gamma x_{1} \cos \omega t\right.$

$\left\{m_{2} \ddot{x}_{2}+\tilde{c}_{2}\left(x_{2}-x_{1}\right)=0\right.$

где $\boldsymbol{\varepsilon}$ - малый параметр, формально вводимый, для указания малых величин в уравнениях, после проведения выкладок положим его равным 1 Порождающая система, получающаяся из (3), при $\gamma=R_{c k}=0$ имеет характеристикой уравнение:

$$
P^{4}+\left(\frac{c_{01}+c_{02}}{m_{1}}+\frac{c_{02}}{m_{2}}\right) P^{2}+\frac{c_{01} c_{02}}{m_{1} m_{2}}=0
$$

корни которого дают две собственные частоты колебаний

$$
\begin{gathered}
\omega_{1}=\sqrt{\frac{1}{2}\left(\frac{c_{1}+c_{2}}{m_{1}}+\frac{c_{2}}{m_{2}}\right)-\sqrt{\frac{1}{4}\left(\frac{c_{1}+c_{2}}{m_{1}}+\frac{c_{2}}{m_{2}}\right)^{2}-\frac{c_{1} c_{2}}{m_{1} m_{2}}}} \\
\omega_{2}=\sqrt{\frac{1}{2}\left(\frac{c_{1}+c_{2}}{m_{1}}+\frac{c_{2}}{m_{2}}\right)+\sqrt{\frac{1}{4}\left(\frac{c_{1}+c_{2}}{m_{1}}+\frac{c_{2}}{m_{2}}\right)^{2}-\frac{c_{1} c_{2}}{m_{1} m_{2}}}}
\end{gathered}
$$

Общее решение порождающей системы записывается так

$\left\{x_{1}=a_{11} \sin \left(\omega_{2} t+\alpha_{1}\right)+a_{12} \sin \left(\omega_{2} t+\alpha_{2}\right)\right.$

$\left\{x_{2}=a_{21} \sin \left(\omega_{1} t+\alpha_{1}\right)+a_{22} \sin \left(\omega_{2} t+\alpha_{2}\right)\right.$

где амплитуды, связаны соотношениями

$$
\begin{gathered}
\eta_{21}=\frac{a_{21}}{a_{11}}=\frac{c_{1}+c_{2}-m_{1} \omega_{1}^{2}}{c_{2}}, \\
\eta_{22}=\frac{a_{22}}{a_{12}}=\frac{c_{1}+c_{2}-m_{2} \omega_{2}^{2}}{c_{2}} .
\end{gathered}
$$

Проведем в (3) преобразование к нормальным координатам по формулам

$$
\left\{\begin{array}{l}
x_{1}=a_{11} f_{1}+a_{12} f_{2} \\
x_{2}=a_{21} f_{1}+a_{22} f_{2}
\end{array}\right.
$$

Поскольку $a_{11}$ и $a_{12}$ - произвольные числа, примем их числами равными индексами 1 , так что в результате преобразования имеем:

$$
\left\{\begin{array}{l}
\ddot{f}_{1}+\omega_{1}^{2} f_{1}=\varepsilon \delta_{1}\left(f_{1}+f_{2}\right) \cos \omega t \\
\ddot{f}_{2}+\omega_{2}^{2} f_{2}=\varepsilon \delta_{2}\left(f_{1}+f_{2}\right) \cos \omega t
\end{array}\right.
$$

где

$$
\delta_{1}=\frac{c_{1} \gamma}{m_{1}+m_{2} \eta_{21}^{2}}, \delta_{2}=\frac{c_{1} \gamma}{m_{1}+m_{2} \eta_{22}^{2}} .
$$

Пусть $\omega=\omega^{0}+\varepsilon \delta$, где $\omega^{0}$ некоторая характерная постоянная, $\lambda$-расстояния. Перепишем систему (9) в виде

$\left\{\begin{array}{l}\dot{f}_{1}=z_{1}, \dot{z}_{1}+\omega_{1}^{2} f_{1}=\varepsilon \delta_{1}\left(f_{1}+f_{2}\right) \cos \omega t \\ \dot{f}_{2}=z_{2}, \dot{z}_{2}+\omega_{2}^{2} f_{2}=\varepsilon \delta_{2}\left(f_{1}+f_{2}\right) \cos \omega t\end{array}\right.$

и будем искать решение в форме

$f_{j}=a_{j} \sin \psi_{j}, z_{j}=a_{j} \omega_{j} \cos \psi_{j}$

$\psi_{j}=\frac{\omega_{j}}{\omega^{0}} \varphi(t)+\theta_{j}(t), \dot{\varphi}(t)=\omega^{0}+\varepsilon \lambda$;

$i=1,2$

где $a_{1}, a_{2}, \theta_{1}, \theta_{2}$ - новые переменные. Дифференцируя далее выражения (11) по $t$ и вставляя найденные производные в систему уравнений (10), получаем относительно медленно меняющихся, переменных $a_{1}, a_{2}, \theta_{1}, \theta_{2}$ уравнения

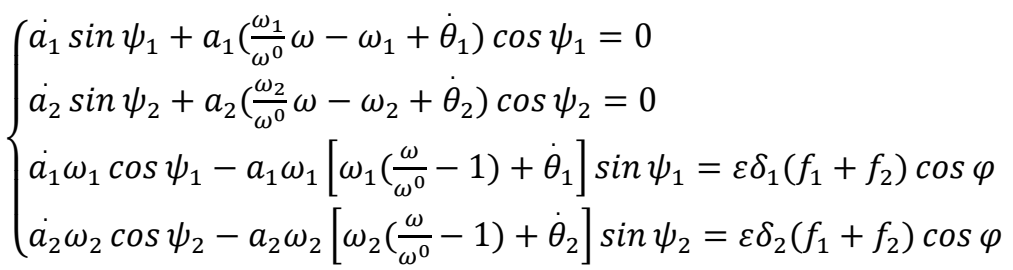

или, разрешая относительно производных,

$$
\left\{\begin{array}{l}
\dot{a}_{1}=\frac{\varepsilon}{\omega_{1}} \delta_{1}\left(a_{1} \sin \psi_{1}+a_{2} \sin \psi_{2}\right) \cos \varphi \cos \psi_{1} \\
\dot{a}_{2}=\frac{\varepsilon}{\omega_{2}} \delta_{2}\left(a_{1} \sin \psi_{1}+a_{2} \sin \psi_{2}\right) \cos \varphi \cos \psi_{2} \\
\dot{\theta_{1}}=\omega_{1}\left(1-\frac{\omega}{\omega^{0}}\right)-\frac{\varepsilon \delta_{1}}{\omega_{1} a_{1}}\left(a_{1} \sin \psi_{1}+a_{2} \sin \psi_{2}\right) \cos \varphi \sin \psi_{1} \\
\dot{\theta_{2}}=\omega_{2}\left(1-\frac{\omega}{\omega^{0}}\right)-\frac{\varepsilon \delta_{2}}{\omega_{2} a_{2}}\left(a_{1} \sin \psi_{1}+a_{2} \sin \psi_{2}\right) \cos \varphi \sin \psi_{2}
\end{array}\right.
$$




\begin{tabular}{|c|c|c|c|c|c|c|}
\hline \multirow{4}{*}{ Impact Factor: } & ISRA (India) & $=3.117$ & SIS (USA) & $=0.912$ & ICV (Poland) & $=6.630$ \\
\hline & ISI (Dubai, UAI & $=0.829$ & РИНЦ (Russia & $=0.156$ & PIF (India) & $=1.940$ \\
\hline & GIF (Australia) & $=0.564$ & ESJI (KZ) & $=8.716$ & IBI (India) & $=4.260$ \\
\hline & JIF & $=1.500$ & SJIF (Morocce & $=5.667$ & OAJI (USA) & $=0.350$ \\
\hline
\end{tabular}

Для приближенного решения системы (13) используем метод усреднения, исходя из предположения, что между частотой пульсаций $\boldsymbol{\omega}$ и частотами собственных колебаний порождающей системы имеется зависимость

$\omega^{0}=\omega_{1}+\omega_{2}$.

Тогда усредняя правые части (13) с учетом (14) по явно входящему времени и, принимая при интегрировании

$\varphi=\omega^{0} t, \theta_{1}=$ const,$\theta_{2}=$ const,

получаем следующие уравнения первого приближения усредненные; для определения области комбинационного резонанса

$$
\left\{\begin{array}{l}
\dot{a}_{1}=\frac{\varepsilon}{4 \omega_{1}} \delta_{1} a_{2} \sin \left(\theta_{1}+\theta_{2}\right) \\
\dot{a}_{2}=\frac{\varepsilon}{4 \omega_{2}} \delta_{2} a_{1} \sin \left(\theta_{1}+\theta_{2}\right) \\
\dot{\theta}_{1}=\omega_{1}\left(1-\frac{\omega}{\omega^{0}}\right)+\frac{\varepsilon a_{2}}{4 \omega_{1} a_{2}} \delta_{1} \cos \left(\theta_{1}+\theta_{2}\right) \\
\dot{\theta}_{2}=\omega_{2}\left(1-\frac{\omega}{\omega^{0}}\right)+\frac{\varepsilon a_{1}}{4 \omega_{2} a_{2}} \delta_{2} \cos \left(\theta_{1}+\theta_{2}\right)
\end{array}\right.
$$

Полагая $\omega^{0}=2 \omega_{1}$ результате усреднения получим систему, уравнений для определения границ области главного параметрического резонанса

$$
\left\{\begin{array}{l}
\dot{a_{1}}=\frac{1}{4} \frac{\varepsilon}{\omega_{1}} \delta_{1} a_{1} \sin 2 \theta_{1} \\
\dot{\theta}_{1}=\omega_{1}\left(1-\frac{\omega}{\omega^{0}}\right)+\frac{1}{4} \frac{\varepsilon \delta_{1}}{\omega_{1}} \cos 2 \theta_{1}
\end{array}\right.
$$

Причем $\dot{a}_{2}=0, \dot{\theta}_{1}=0$. Эти же уравнения получаются из (16) после замены всех индексов 2 на 1. Система уравнений для определения границ областей главного параметрического резонанса $2 \omega_{2}$ получается из (17) заменой всех индексов 1 на 2, но следует прибавить $\dot{a}_{1}=0, \dot{\theta}_{1}=0$.

Введем обозначения

$$
\left\{\begin{array}{l}
l_{1}=\frac{\varepsilon \delta_{1}}{4 \omega_{1}}, d_{1}=\omega_{1}\left(1-\frac{\omega}{\omega^{0}}\right) \\
l_{2}=\frac{\varepsilon \delta_{2}}{4 \omega_{2}}, d_{2}=\omega_{2}\left(1-\frac{\omega}{\omega^{0}}\right)
\end{array}\right.
$$

и новые переменные

$$
\left\{\begin{array}{l}
u_{1}=a_{1} \sin \theta_{1}, u_{1}=a_{1} \cos \theta_{1} \\
u_{2}=a_{2} \sin \theta_{2}, u_{2}=a_{2} \cos \theta_{2}
\end{array}\right.
$$

Тогда, дифференцируя (19) и делая элементарные преобразования, получаем из (16) систему 4-х линейных дифференциальных уравнений с постоянными коэффициентами:

$$
\begin{gathered}
\dot{u}_{1}=d_{1} v_{1}+l_{1} v_{2}, \dot{v}_{1}=-d_{1} u_{1}+l_{1} u_{2} \\
\dot{u}_{2}=d_{2} v_{2}+l_{2} v_{1}, \dot{v}_{2}=-d_{2} u_{2}+l_{2} u_{1}
\end{gathered}
$$

Характеристическое уравнение системы (20) имеет вид

$$
\rho^{4}-\left(2 l_{1} l_{2}-d_{1}^{2}-d_{2}^{2}\right) \rho^{2}+\left(d_{1} d_{2}-l_{1} l_{2}\right)^{2}=0
$$

корни которого

$$
\rho= \pm \sqrt{\frac{1}{2}\left[2 l_{1} l_{2}-d_{1}^{2}-d_{2}^{2} \pm\left(d_{2}-d_{1}\right) \sqrt{\left(d_{1}+d_{2}\right)^{2}-4 l_{1} l_{2}}\right]}
$$

Очевидно, величины $u_{1}, v_{1}, u_{2}, v_{2}$ а, следовательно, и $a_{1}, a_{2}$ будут неограниченно возрастать при $t \rightarrow \infty$, если вещественная часть хотя бы одного характеристического числа (22) положительна. Применительно к (22) невозмущенное движение системы неустойчиво при условии

$$
4 l_{1} l_{2}>\left(d_{1}+d_{2}\right)^{2},
$$

откуда имеем границы области комбинационного резонанса $\varepsilon=1$

$\omega_{1}+\omega_{2}-\frac{1}{2} \frac{\sqrt{\delta_{1} \delta_{2}}}{\sqrt{\omega_{1} \omega_{2}}}<\omega<\omega_{1}+\omega_{2}+\frac{1}{2} \frac{\sqrt{\delta_{1} \delta_{2}}}{\sqrt{\omega_{1} \omega_{2}}}$

заменяя все индексы 2 на 1 , а затем 1 на 2 , получаем из (24) выражения для построения границ двух областей главного параметрического резонанса

$$
\begin{aligned}
& 2 \omega_{1}-\frac{\delta_{1}}{2 \omega_{1}}<\omega<2 \omega_{1}+\frac{\delta_{1}}{2 \omega_{1}}, \\
& 2 \omega_{2}-\frac{\delta_{2}}{2 \omega_{2}}<\omega<2 \omega_{2}+\frac{\delta_{2}}{2 \omega_{2}} .
\end{aligned}
$$

Введем обозначения: $\Omega_{1}=\sqrt{\frac{c_{1}}{m_{1}}}$ собственная частота основной системы при отсутствии гасителя, $\Omega_{2}=\sqrt{\frac{c_{2}}{m_{2}}}$ - парциальная частота гасителя, $\mu=\frac{m_{2}}{m_{1}}$ масс гасителя и основной системы. Рассмотрим построение областей параметрического и комбинационного резонанса в параметрах $\gamma, \omega$. При $\boldsymbol{\gamma} \rightarrow \mathbf{0}$ из (24)(26) получаем расположение начал областей главного параметрического резонанса $\left[2 \omega_{1}\right],\left[2 \omega_{2}\right]$ и комбинационного резонанса $\left[\omega_{1}+\right.$ $\boldsymbol{\omega}_{2}$ ] соответственно. При отсутствии динамического гасителя $\left(\boldsymbol{m}_{\mathbf{2}}=\mathbf{0}\right)$ собственная частота основной системы $\Omega$, и имеем одну область главного параметрического резонанса $2 \Omega_{1}$. Поскольку согласно (5)

$$
\left(\omega_{1}+\omega_{2}\right)^{2}=\frac{c_{1}+c_{2}}{m_{1}}+\frac{c_{2}}{m_{2}}+2 \sqrt{\frac{c_{1} c_{2}}{m_{1} m_{2}}},
$$

то нетрудно убедиться, что, вообще говоря, может быть

или

$$
\omega_{1}+\omega_{2}=2 \Omega_{1}
$$

$$
\frac{c_{1}+c_{2}}{m_{1}}+\frac{c_{2}}{m_{2}}+2 \sqrt{\frac{c_{1} c_{2}}{m_{1} m_{2}}}=4 \frac{c_{1}}{m_{1}},
$$

т.е. присоединение динамического гасителя не устранит области главного параметрического резонанса системы без гасителя. Действительно, так как

$$
\frac{c_{2}}{m_{1}}=\frac{c_{2}}{m_{2}} \frac{m_{2}}{m_{1}}=\mu \Omega_{1}^{2},
$$

то (27) переписывается как

$$
\Omega_{1}^{2}+\mu \Omega_{2}^{2}+\Omega_{2}^{2}+2 \Omega_{1} \Omega_{2}=4 \Omega_{1}^{2}
$$

или

$$
(1+v)^{2}+\mu v^{2}=4,
$$

где $v=\frac{\Omega_{1}}{\Omega_{2}}$ - настройка гасителя, откуда 


\begin{tabular}{|c|c|c|c|c|c|c|}
\hline \multirow{4}{*}{ Impact Factor: } & ISRA (India) & $=3.117$ & SIS (USA) & $=0.912$ & ICV (Poland) & $=6.630$ \\
\hline & ISI (Dubai, UAE & $=0.829$ & РИНЦ (Russia) & $=0.156$ & PIF (India) & $=1.940$ \\
\hline & GIF (Australia) & $=0.564$ & ESJI (KZ) & $=8.716$ & IBI (India) & $=4.260$ \\
\hline & JIF & $=1.500$ & SJIF (Morocco) & $=5.667$ & OAJI (USA) & $=0.350$ \\
\hline$=\frac{-1 \pm \sqrt{4+3 \mu}}{1+\mu}$. & & & Для & тва & ний & дем \\
\hline
\end{tabular}
соответствующее знаку "+" так что при малом $\mu$ можно записать

$$
\begin{gathered}
v_{1}=\frac{2 \sqrt{1+\frac{3}{4} \mu-1}}{1+\mu} \approx \frac{1+\frac{3}{4} \mu}{1+\mu} . \\
\frac{\omega_{1}}{\Omega_{1}}=\sqrt{\frac{1}{2}\left[1+(1+\mu) v^{2}\right]-\sqrt{\frac{1}{4}\left[1+(1+\mu) v^{2}\right]^{2}-v^{2}}}, \frac{2 \omega_{2}}{\Omega_{2}}, \frac{\omega_{1}+\omega_{2}}{\Omega_{1}} \\
\frac{\omega_{2}}{\Omega_{1}}=\sqrt{\frac{1}{2}\left[1+(1+\mu) v^{2}\right]+\sqrt{\frac{1}{4}\left[1+(1+\mu) v^{2}\right]^{2}-v^{2}}} .
\end{gathered}
$$

\section{Таблица 1}

\begin{tabular}{|c|l|l|l|}
\hline \multicolumn{4}{|c|}{$\mu=0.05$} \\
\hline$v$ & $\frac{2 \omega_{1}}{\Omega_{1}}$ & $\frac{2 \omega_{2}}{\Omega_{1}}$ & $\frac{\omega_{1}+\omega_{2}}{\Omega_{1}}$ \\
\hline 0,5 & 1,183 & 2,027 & 1,605 \\
\hline 0,6 & 1,368 & 2,045 & 1,707 \\
\hline 0,7 & 1,540 & 2,077 & 1,808 \\
\hline 0,8 & 1,685 & 2,135 & 1,910 \\
\hline 0,9 & 1,788 & 2,236 & 2,012 \\
\hline 1,0 & 1,848 & 2,379 & 2,114 \\
\hline 1,1 & 1,881 & 2,550 & 2,216 \\
\hline 1,2 & 1,900 & 2,736 & 2,318 \\
\hline 1,3 & 1,912 & 2,928 & 2,420 \\
\hline 1,4 & 1,920 & 2,124 & 2,522 \\
\hline
\end{tabular}

\begin{tabular}{|c|l|l|l|}
\hline \multicolumn{4}{|c|}{$\mu=0.1$} \\
\hline$v$ & $\frac{2 \omega_{1}}{\Omega_{1}}$ & $\frac{2 \omega_{2}}{\Omega_{1}}$ & $\frac{\omega_{1}+\omega_{2}}{\Omega_{1}}$ \\
\hline 0,5 & 1,168 & 2,053 & 1,611 \\
\hline 0,6 & 1,341 & 2,087 & 1,714 \\
\hline 0,7 & 1,495 & 2,140 & 1,817 \\
\hline 0,8 & 1,619 & 2,222 & 1,921 \\
\hline 0,9 & 1,708 & 2,341 & 2,024 \\
\hline 1,0 & 1,766 & 2,490 & 2,128 \\
\hline 1,1 & 1,803 & 2,661 & 2,232 \\
\hline 1,2 & 1,827 & 2,845 & 2,336 \\
\hline 1,3 & 1,843 & 3,037 & 2,440 \\
\hline 1,4 & 1,855 & 3,233 & 2,544 \\
\hline
\end{tabular}

\begin{tabular}{|c|c|l|l|}
\hline \multicolumn{4}{|c|}{$\mu=0.2$} \\
\hline$v$ & $\frac{2 \omega_{1}}{\Omega_{1}}$ & $\frac{2 \omega_{2}}{\Omega_{1}}$ & $\frac{\omega_{1}+\omega_{2}}{\Omega_{1}}$ \\
\hline 0,5 & 1,140 & 2,104 & 1,622 \\
\hline 0,6 & 1,294 & 2,162 & 1,728 \\
\hline 0,7 & 1,426 & 2,245 & 1,835 \\
\hline 0,8 & 1,527 & 2,356 & 1,942 \\
\hline 0,9 & 1,602 & 2,496 & 2,049 \\
\hline 1,0 & 1,654 & 2,658 & 2,156 \\
\hline 1,1 & 1,691 & 2,837 & 2,264 \\
\hline 1,2 & 1,718 & 3,026 & 2,372 \\
\hline 1,3 & 1,737 & 3,223 & 2,480 \\
\hline 1,4 & 1,751 & 3,425 & 2,588 \\
\hline
\end{tabular}




\begin{tabular}{|c|c|c|c|c|c|c|}
\hline \multirow{4}{*}{ Impact Factor: } & ISRA (India) & $=3.117$ & SIS (USA) & $=0.912$ & ICV (Poland) & $=6.630$ \\
\hline & ISI (Dubai, UAI & $=0.829$ & РИНЦ (Russia & $=0.156$ & PIF (India) & $=1.940$ \\
\hline & GIF (Australia) & $=0.564$ & ESJI (KZ) & $=8.716$ & IBI (India) & $=4.260$ \\
\hline & JIF & $=1.500$ & SJIF (Morocce & $=5.667$ & OAJI (USA) & $=0.350$ \\
\hline
\end{tabular}

\section{Выводы.}

Применительно к техническим приложениям можно попытаться подобрать настройку гасителя так, чтобы начала областей неустойчивости, определяемых выражениями (24)-(26) находились как можно дальше от точки, $\omega=2 \Omega$ что можно рассматривать как оптимальное расположение начал областей неустойчивости в системе с гасителем. В таблице 1 приведены безразмерные абсциссы расположения начал областей неустойчивости для разных настроек гасителя. Видим, что наиболее подходящей, например, при $\mu=0.1$ является настройка $v=1.1$.

\section{References:}

1. Panovko, Y. G. (1967). Osnovi prikladnoy teorii uprugix kolebaniy (p.316). Moskva: Mashinostroyeniye.

2. Rjanitsin, A. R. (1968). Teoriya polzuchesti. (p. 415). Moskva: Stroyizdat.

3. Osetinskiy, Y. V. (1967). K voprosu o kombinasionnom parametricheskom rezonanse v uprugix sistemax. Prikladnaya mexanika, AN USSR, 3(18), pp. 113-118.

4. Koltunov, M. A. (1976). Polzuchest $i$ relaksasiya. (p. 277). Moskva: Visshaya skola.

5. (1981). Vibrasii v texnike: Spravochnik: V 6 t. t.6. Zashita ot vibrasii i udarov. Pod red. K.V. Frolova (Eds.). (p.456). Moskva: Mashinostroyeniye.

6. Tokarev, M. F., Taliskiy, Y. N., \& Frolov, V. A. (1984). Mexanicheskiye vozdeystviya $i$ zashita radioelektronnoy apparaturi: Ucheb. posobiye dlya vuzov (p.224). Pod red. V.A. Frolova (Eds.). Moskva: Radio i svyaz.
7. Nashif, A., Djouns, D., \& Xenderson, D. (1988). Dempfirovaniye kolebaniy. Per. s angl. (p.448). Moskva: Mir.

8. Ilinskiy, V. S. (1982). Zashita REA $i$ presizionnogo oborudovaniya ot dinamicheskix vozdeystviy. (p.296). Moskva: Radio i svyaz.

9. Safarov, I. I., Teshayev, M. N., Majidov, M. (2014). Dempfirovaniye kolebaniy mexanicheskix sistem. (p.97). LAP LAMBERT Academic Publishing.

10. Safarov, I. I. (1992). Kolebaniya $i$ volni $v$ dissipativno neodnorodnix sredax $i$ konstruksiyax. (p.252). Tashkent: Fan.

11. Rashidov, T. R. (1973). Dinamicheskaya teoriya Seys mostoykosti slojnix sistem podzemnix soorujeniy. (p.182). Tashkent: Fan.

12. Mau, M. (1963). Dinamicheskiye napryajeniya i smeshyeniye $\mathrm{v}$ blizi silindricheskoy poverxnosti razriva ot ploskoy garmonicheskoy volni sdviga. Prikladnaya mexanika, perevod s angliyskogo, t.30, ser Ye, № 3, pp.117-126. 